\title{
List of Figures:
}

Figure 1. A schematic representation of the synthesized $\mathrm{Zr}-\mathrm{Sn}$ /graphene nanocomposite catalyst.

Figure 2. Schematic of a CHFS reactor set-up used for the synthesis of $\mathrm{Zr}$-Sn/graphene nanocomposite catalyst.

Figure 3. Transmission Electron Microscopy (TEM) images of (a) pure metal of tin doped zirconium oxide ( $\mathrm{Zr}-\mathrm{Sn}-\mathrm{O})$ (b) tin doped zirconia/ graphene nanocomposite ( $\mathrm{Zr}-\mathrm{Sn} / \mathrm{GO})$ (c) graphene oxide (GO) sample.

Figure 4. X-ray powder diffraction (XRD) patterns of tin doped zirconium oxide ( $\mathrm{Zr}-\mathrm{Sn}-\mathrm{O})$ (b) tin doped zirconia/ graphene nanocomposite ( $\mathrm{Zr}-\mathrm{Sn} / \mathrm{GO})$.

Figure 5. X-ray photoelectron spectroscopy (XPS) spectra showing (a) deconvoluted C(1s), (b) $\mathrm{Zr}$ (3d) region, (c) $\mathrm{Sn}(3 \mathrm{~d})$ region and (d) Sn Auger parameter region.

Figure 6. Model predicted DMC yield $v s$ experimentally obtained DMC yield.

Figure 7. Reaction scheme for the synthesis of $\mathrm{DMC}$ from $\mathrm{PC}$ and $\mathrm{MeOH}$.

Figure 8. Effect of different heterogeneous catalysts on the direct synthesis of DMC.

Experimental conditions: $\mathrm{MeOH}: \mathrm{PC}$ molar ratio 1:1; catalyst loading 2.5\% $(\mathrm{w} / \mathrm{w})$; reaction temperature $433 \mathrm{~K}$; reaction time $4 \mathrm{~h}$ and stirring speed $300 \mathrm{rpm}$.

Figure 9. Effect of $\mathrm{MeOH}: \mathrm{PC}$ molar ratio on the synthesis of DMC. Experimental conditions: Catalyst: Zr-Sn/GO; catalyst loading 2.5\% (w/w); reaction temperature $433 \mathrm{~K}$; reaction time $4 \mathrm{~h}$ and stirring speed $300 \mathrm{rpm}$.

Figure 10. Effect of catalyst loading on PC conversion and yield of DMC. Experimental conditions: Catalyst: $\mathrm{Zr}-\mathrm{Sn} / \mathrm{GO}$; $\mathrm{MeOH}: \mathrm{PC}$ molar ratio 10:1; reaction temperature $433 \mathrm{~K}$; reaction time $4 \mathrm{~h}$ and stirring speed $300 \mathrm{rpm}$.

Figure 11. Effect of reaction temperature on PC conversion and yield of DMC. Experimental conditions: Catalyst: $\mathrm{Zr}-\mathrm{Sn} / \mathrm{GO}$; $\mathrm{MeOH}: \mathrm{PC}$ molar ratio 10:1; catalyst loading 2.5\% (w/w); reaction time $4 \mathrm{~h}$ and stirring speed $300 \mathrm{rpm}$.

Figure 12. Effect of reaction time on PC conversion and yield of DMC. Experimental conditions: Catalyst: $\mathrm{Zr}-\mathrm{Sn} / \mathrm{GO}$; $\mathrm{MeOH}: \mathrm{PC}$ molar ratio 10:1; catalyst loading 2.5\% (w/w); reaction temperature $433 \mathrm{~K}$ and stirring speed $300 \mathrm{rpm}$.

Figure 13. Effect of stirring speed on PC conversion and yield of DMC. Experimental conditions: Catalyst: $\mathrm{Zr}-\mathrm{Sn} / \mathrm{GO}$; $\mathrm{MeOH}: \mathrm{PC}$ molar ratio 10:1; catalyst loading 2.5\% (w/w); reaction temperature $433 \mathrm{~K}$ and reaction time $4 \mathrm{~h}$. 
Figure 14. Effect of catalyst reusability on PC conversion and yield of DMC. Experimental conditions: Catalyst: $\mathrm{Zr}-\mathrm{Sn} / \mathrm{GO}$; $\mathrm{MeOH}: \mathrm{PC}$ molar ratio 10:1; catalyst loading 2.5\% (w/w); reaction temperature $433 \mathrm{~K}$; reaction time $4 \mathrm{~h}$ and stirring speed $300 \mathrm{rpm}$.

Figure 15. Response surface graph: Effect of $\mathrm{MeOH}: \mathrm{PC}$ molar ratio and catalyst loading (w/w) on DMC yield.

Figure 16. Response surface graph: Effect of reaction temperature and stirring speed on DMC yield.

Figure 17. Response surface graph: Effect of catalyst loading (w/w) and reaction time on DMC yield. 


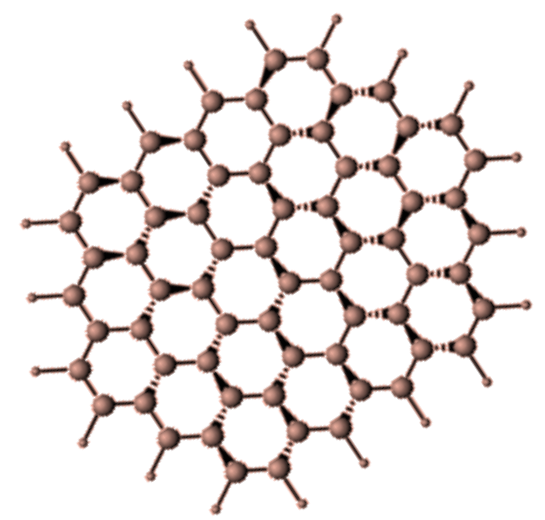

Graphene oxide (GO)

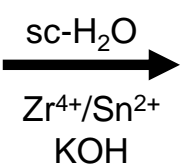

MO: metal oxide $\mathrm{Zr}-\mathrm{Sn}$

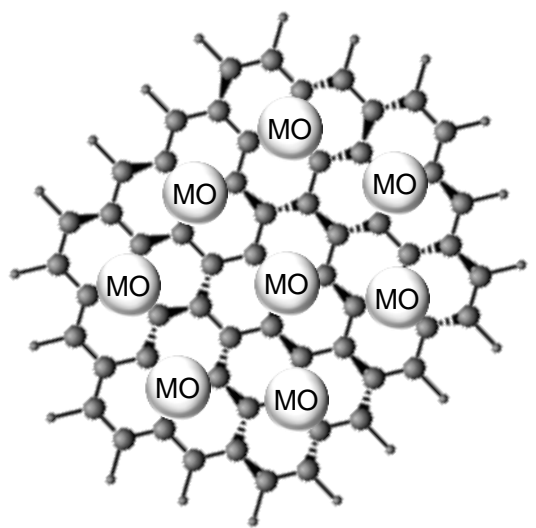

$\mathrm{Sn}-\mathrm{Zr}$ decorated graphene

Figure 1 


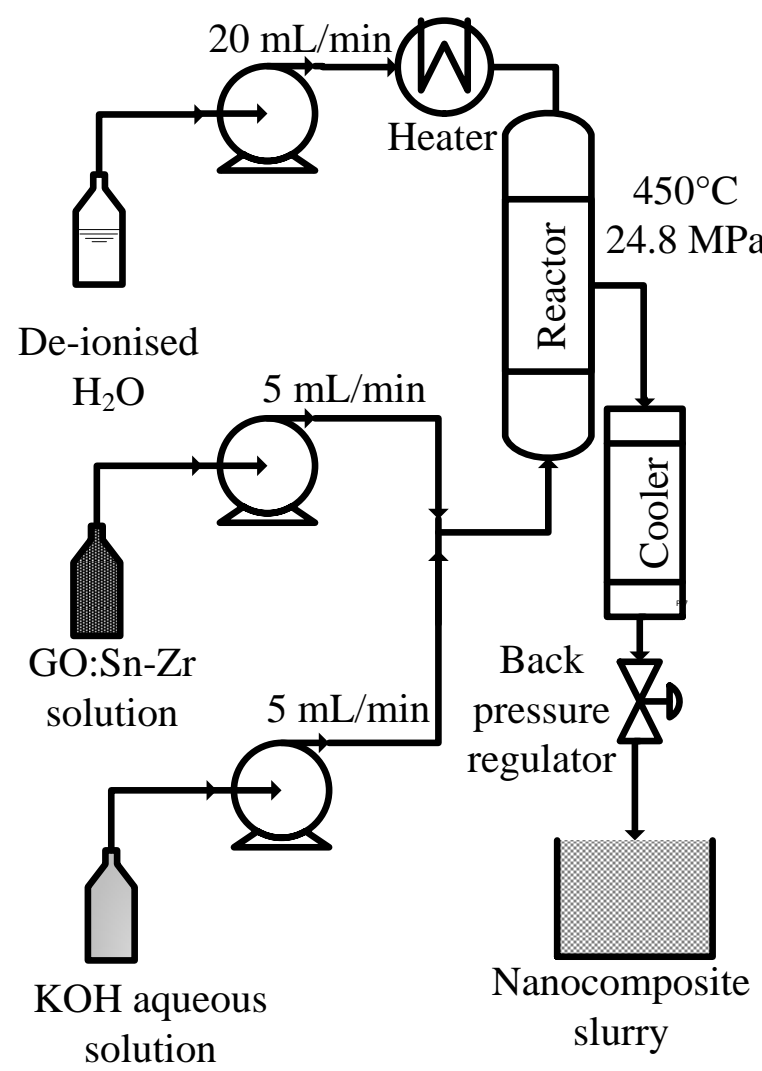

Figure 2 

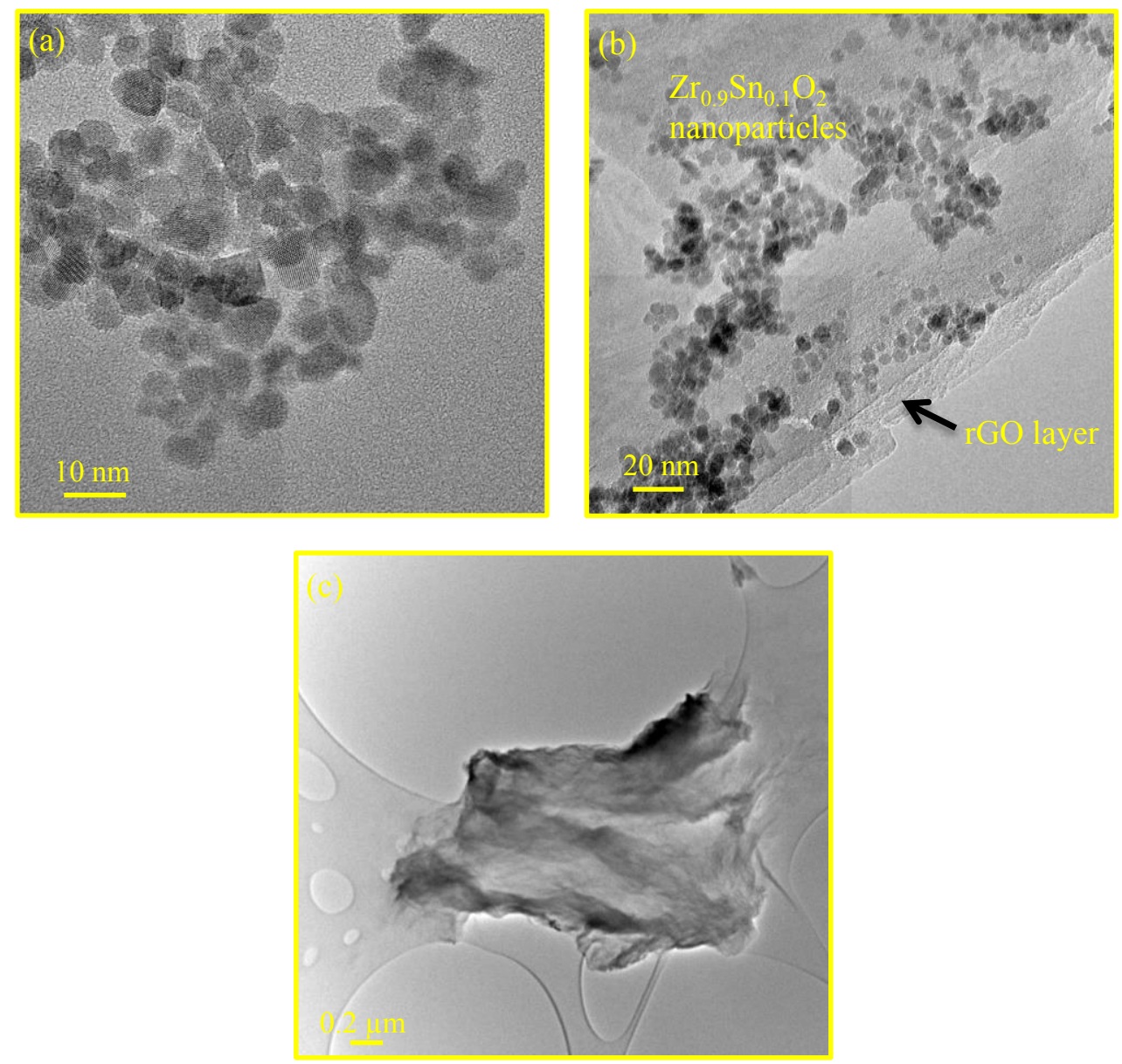

Figure 3 


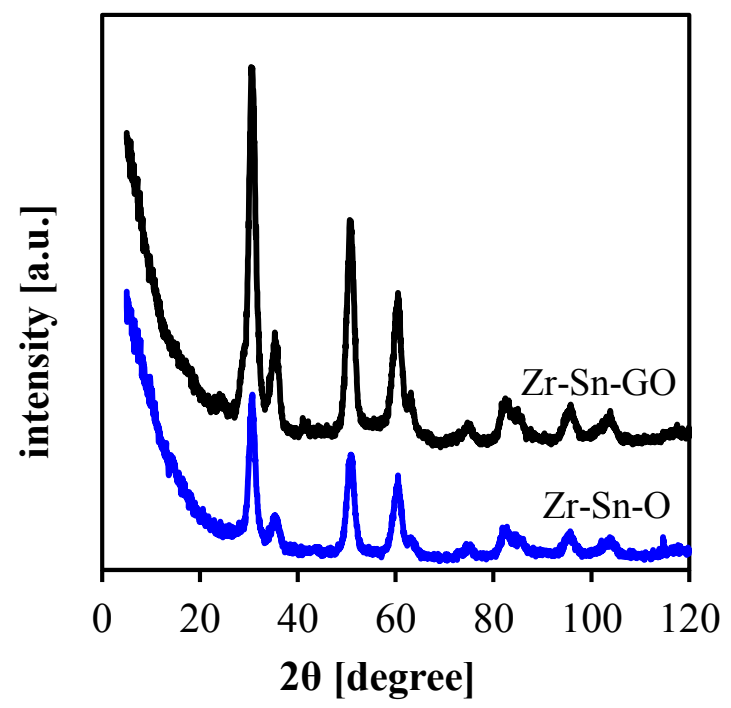

Figure 4 

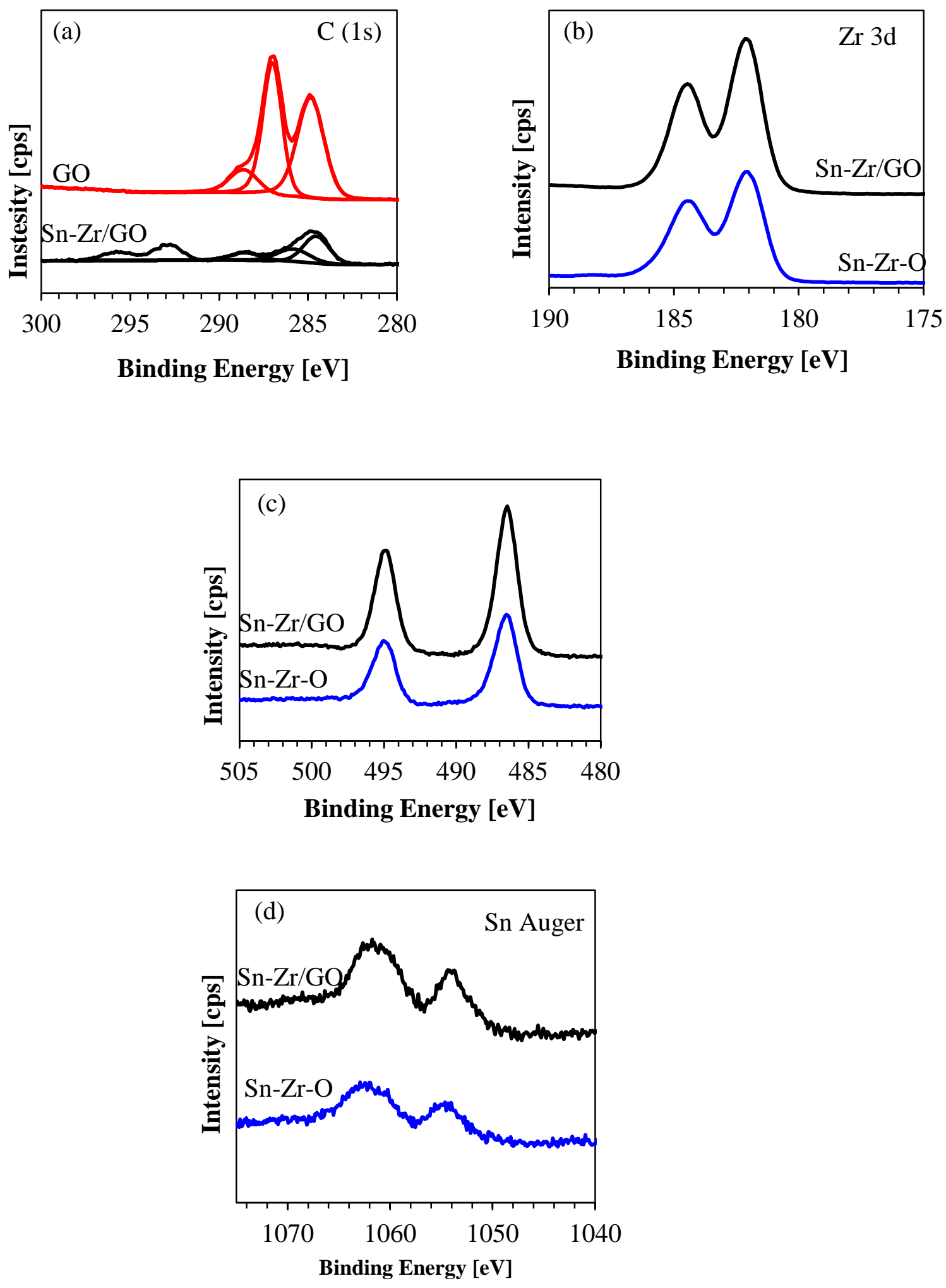

Figure 5 


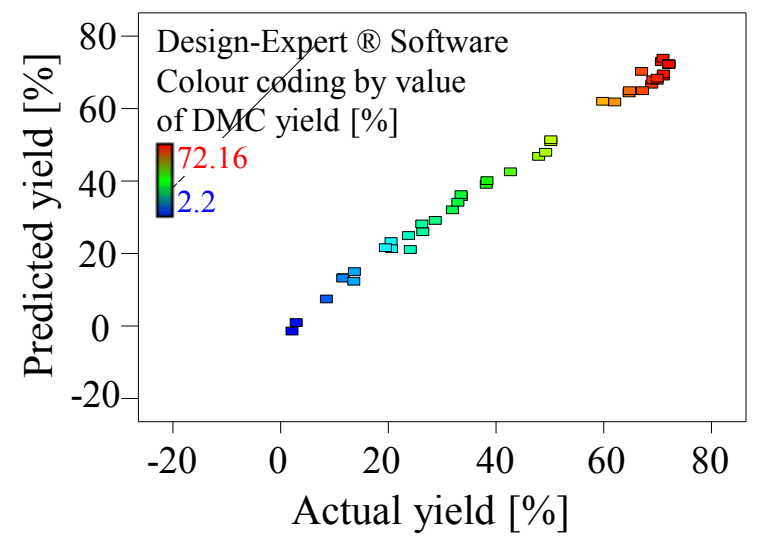

Figure 6 


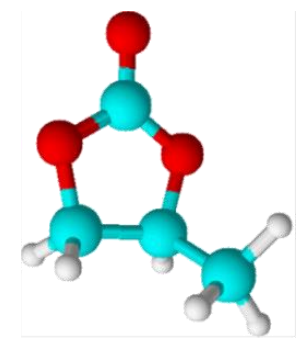

Propylene carbonate

[PC]

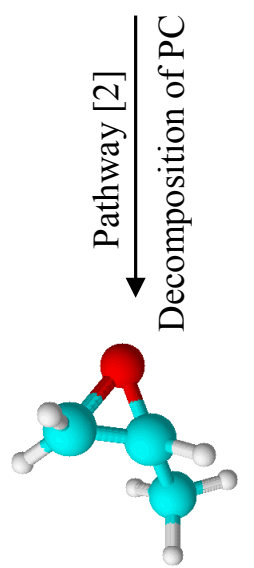

Propylene oxide

[PO]

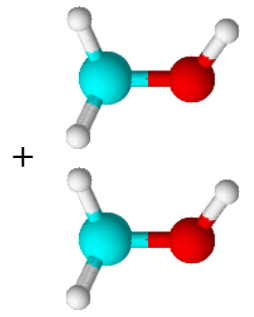

Methanol

[MeOH]

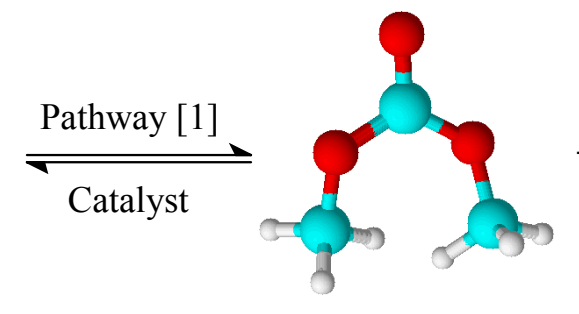

Dimethyl carbonate

[DMC]

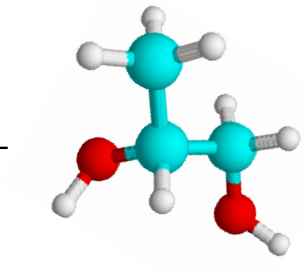

Propylene glycol

[PG]

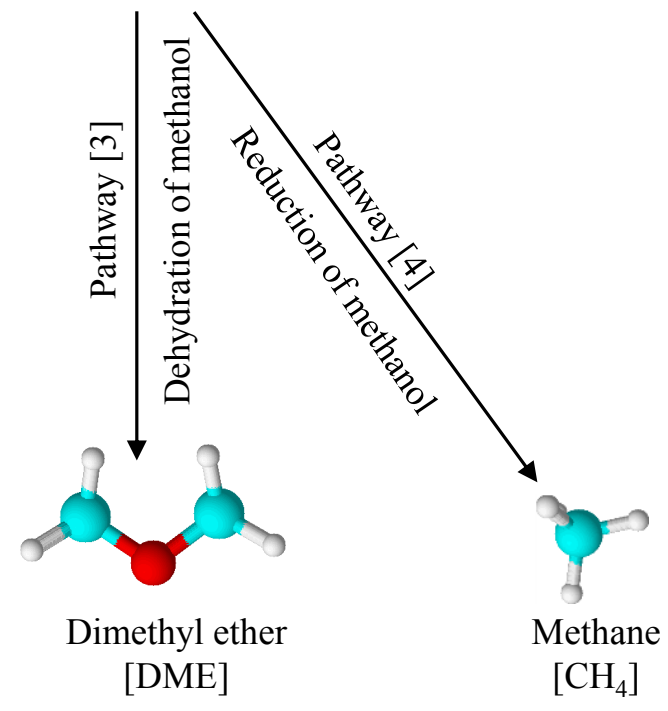

Figure 7 


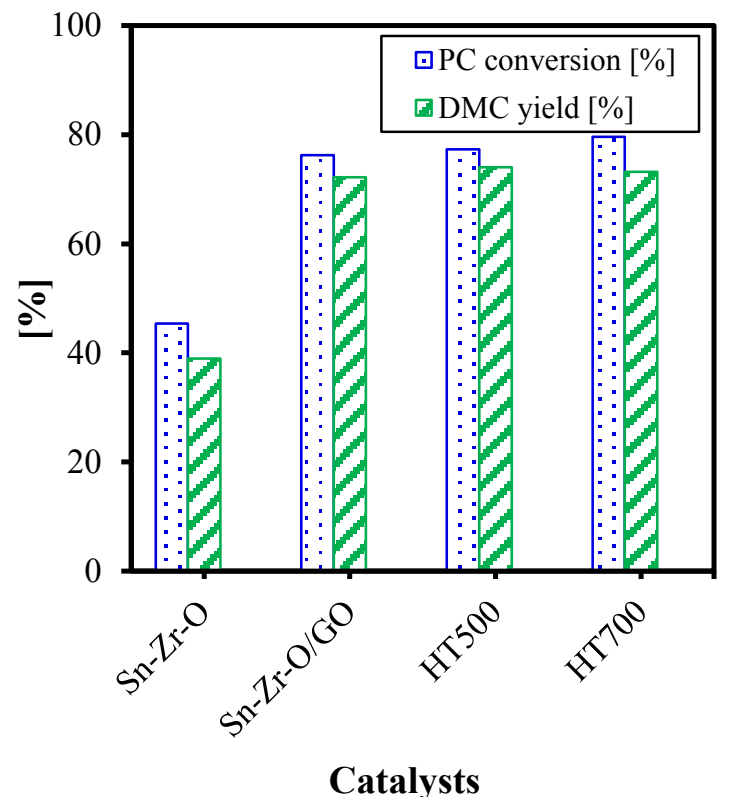

Figure 8 


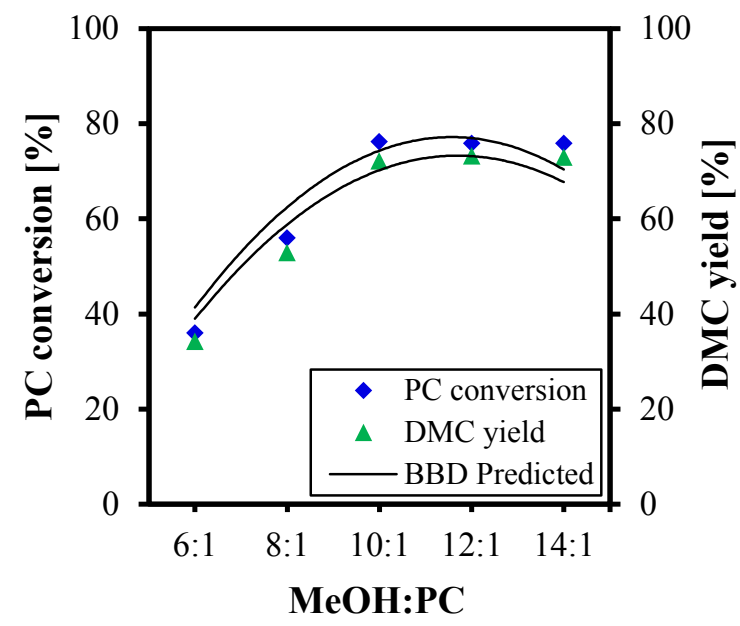

Figure 9 


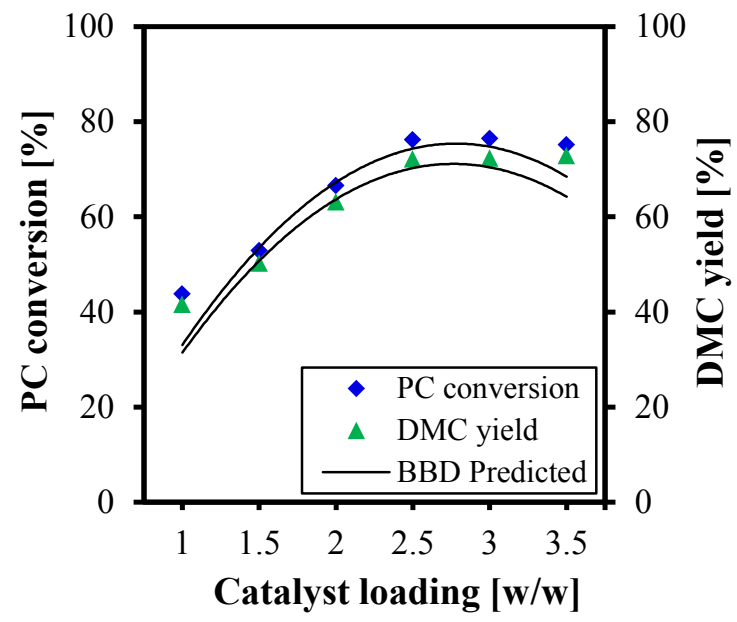

Figure 10 


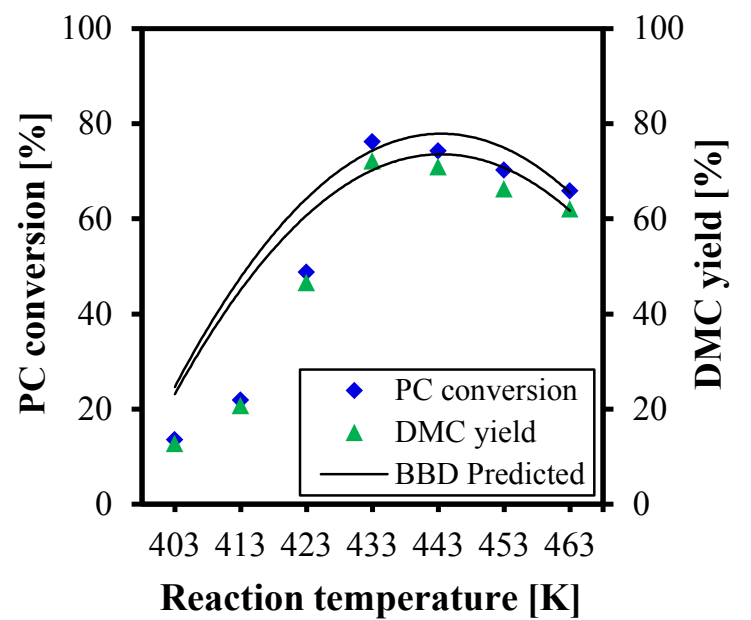

Figure 11 


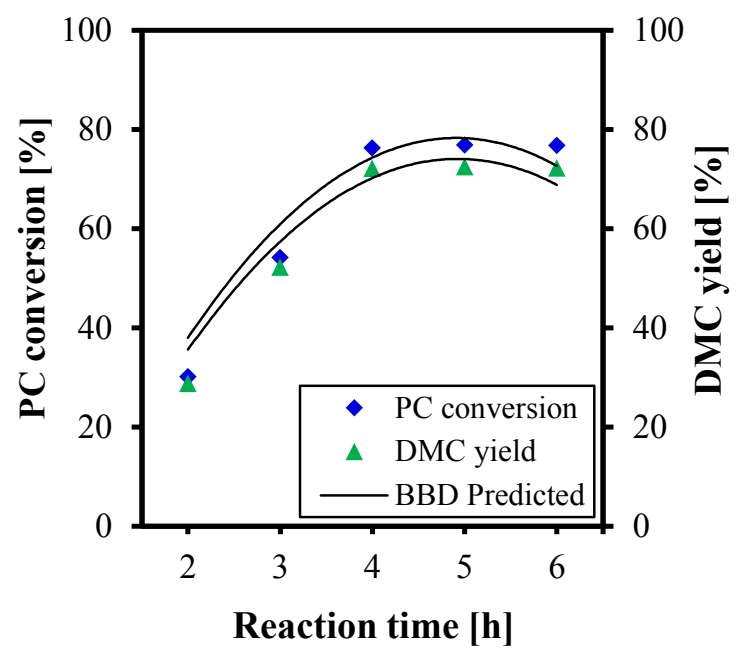

Figure 12 


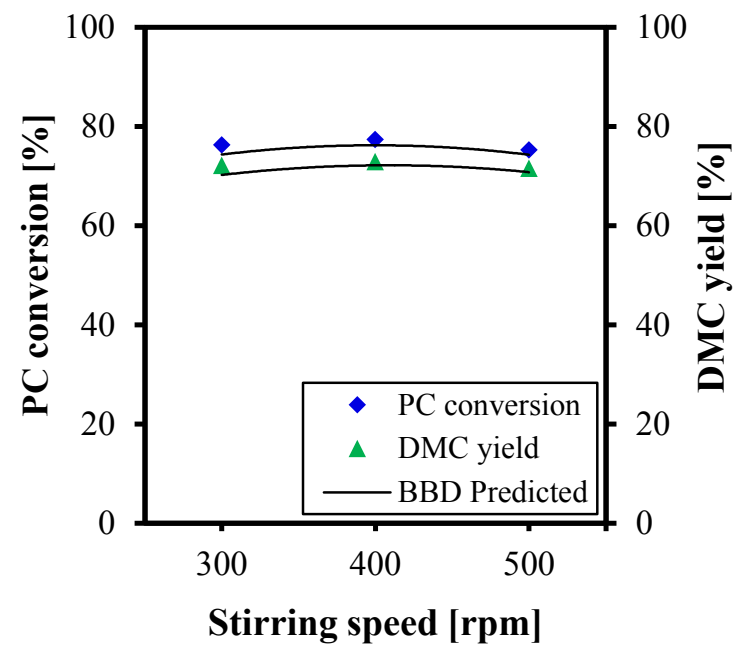

Figure 13 


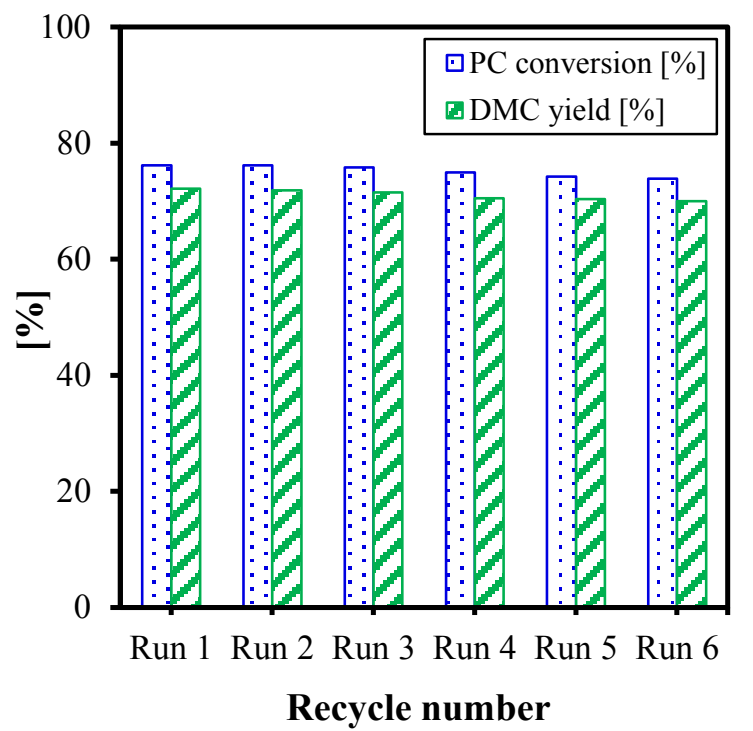

Figure 14 


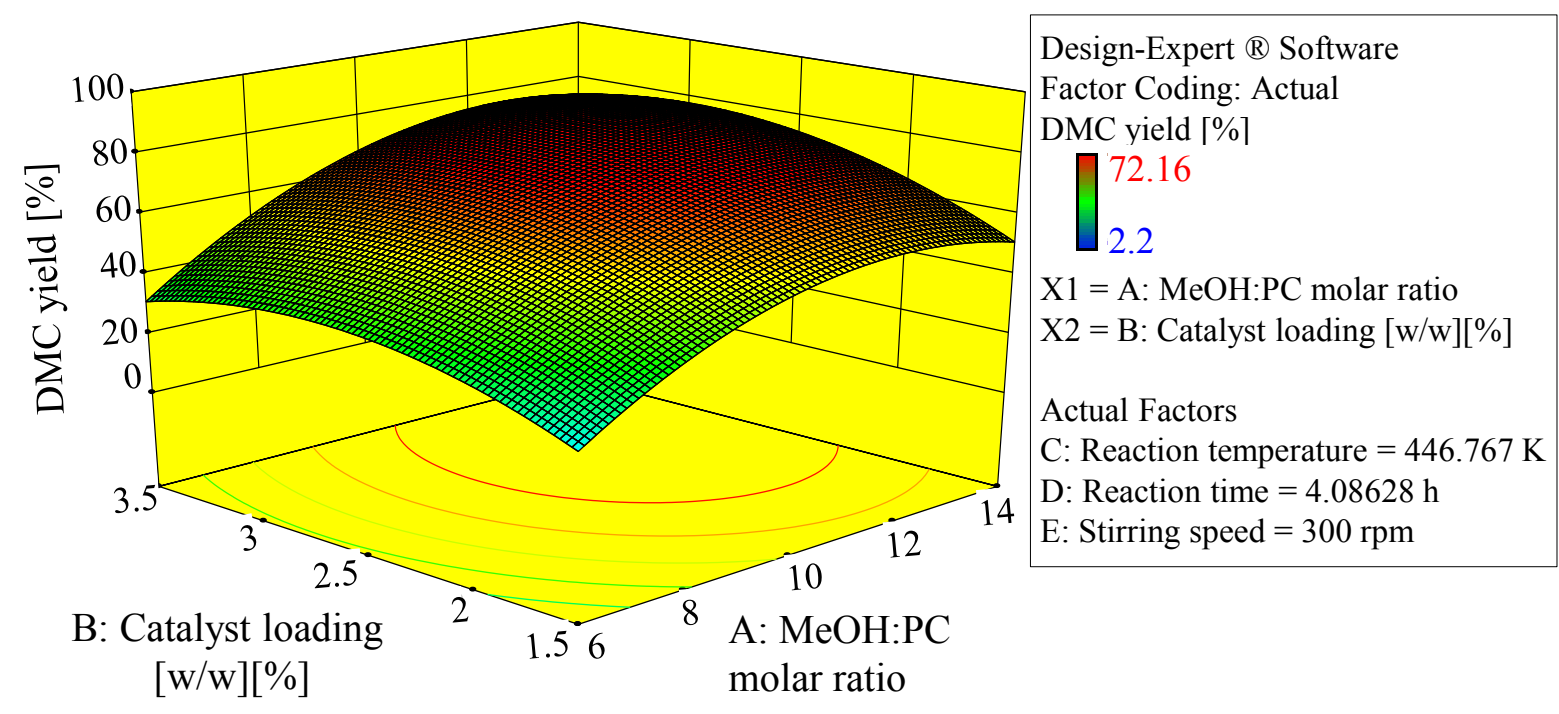

Figure 15 


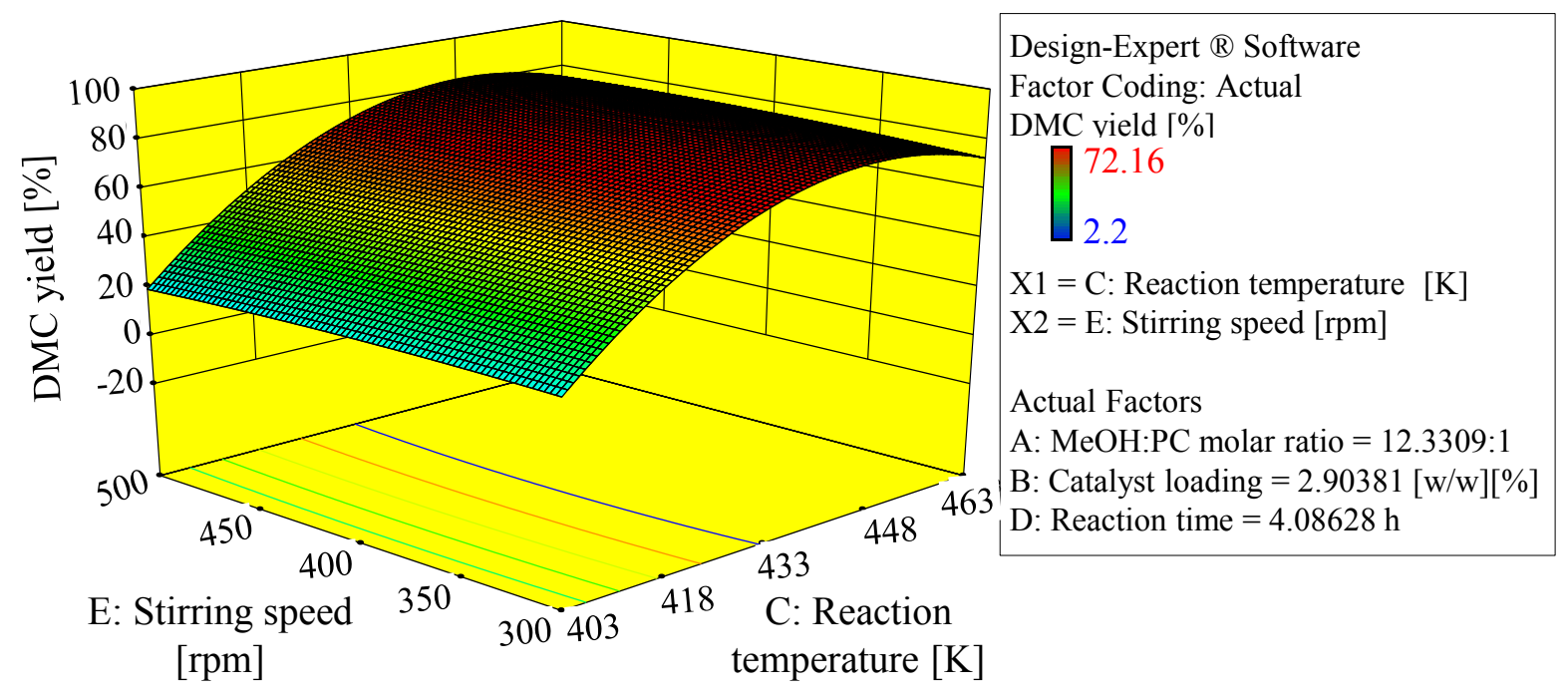

Figure 16 


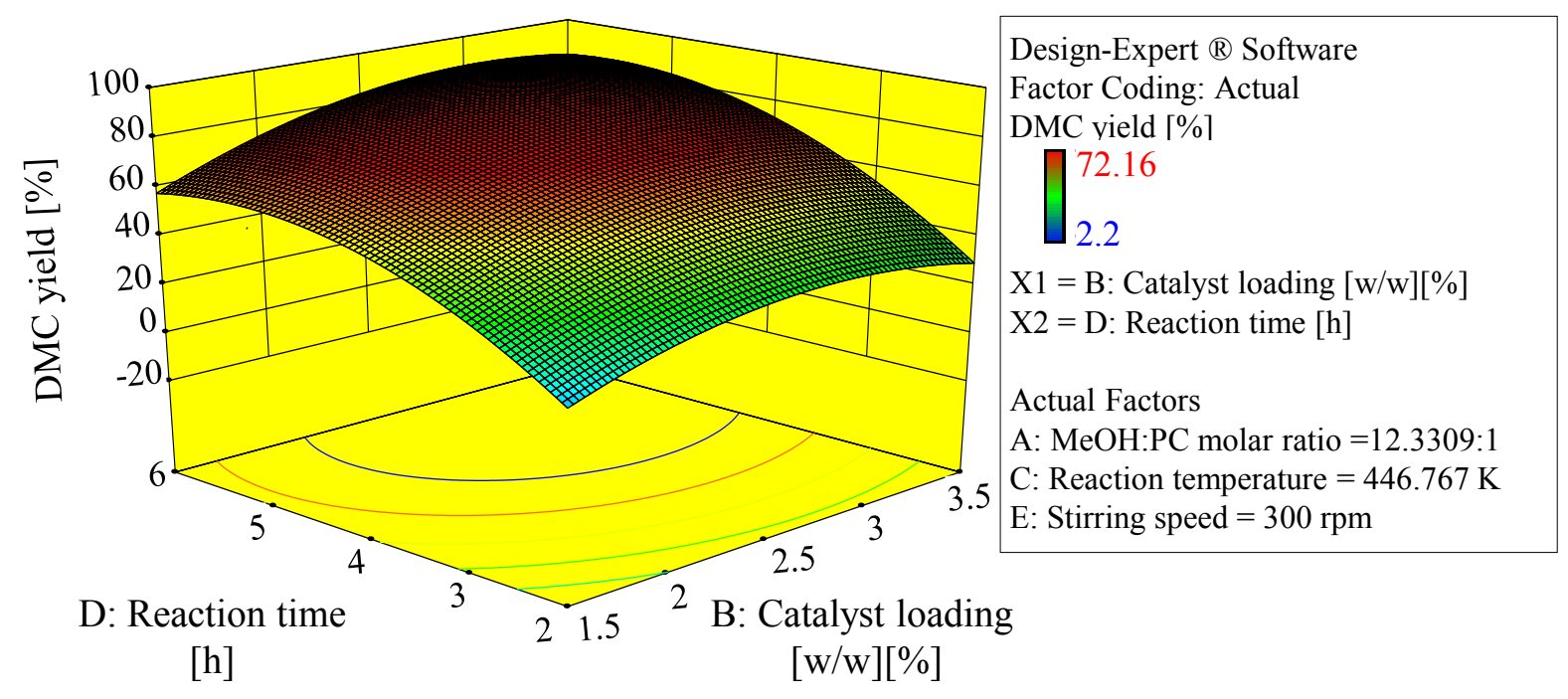

Figure 17 